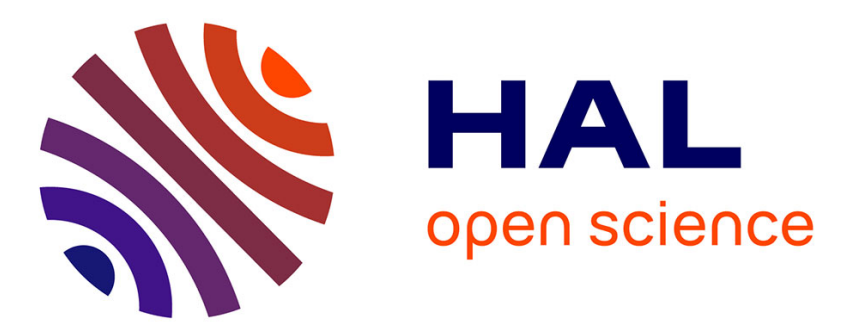

\title{
Les mécanismes de coordination dans les réseaux sociaux : un cadre analytique de la dynamique territoriale
}

\author{
Christophe Boschet, T. Rambonilaza
}

\section{- To cite this version:}

Christophe Boschet, T. Rambonilaza. Les mécanismes de coordination dans les réseaux sociaux: un cadre analytique de la dynamique territoriale. Revue d'économie régionale et urbaine, 2010, 3, p. 569 - p. 593. 10.3917/reru.103.0569 . hal-00556962

\section{HAL Id: hal-00556962 https://hal.science/hal-00556962}

Submitted on 18 Jan 2011

HAL is a multi-disciplinary open access archive for the deposit and dissemination of scientific research documents, whether they are published or not. The documents may come from teaching and research institutions in France or abroad, or from public or private research centers.
L'archive ouverte pluridisciplinaire HAL, est destinée au dépôt et à la diffusion de documents scientifiques de niveau recherche, publiés ou non, émanant des établissements d'enseignement et de recherche français ou étrangers, des laboratoires publics ou privés. 


\title{
Les mécanismes de coordination dans les réseaux sociaux :
}

\author{
un cadre analytique \\ de la dynamique territoriale*
}

\section{Coordination mechanisms in social networks:}

\section{an analytical framework of territorial dynamics}

\author{
Christophe BosCHET** \\ Cemagref, UR Aménités et Dynamiques des Espaces Ruraux \\ 50 avenue de Verdun \\ 33612 CESTAS CEDEX - France \\ Contact : christophe.boschet@cemagref.fr
}

Tina RAmbonilaza

Cemagref, UR Aménités et Dynamiques des Espaces Ruraux

50 avenue de Verdun

33612 CESTAS CEDEX - France

GREThA UMR CNRS 5113

Mots-clés : territoires ruraux, coordination, analyse structurale, dispositifs institutionnels

Keywords : rural areas, coordination, structural analysis, institutional devices

Classification JEL : R58, D85, Q01

*Première version reçue, mars 2009 ; version finale, janvier 2010.

** Les auteurs remercient les relecteurs anonymes, qui, par leurs commentaires et suggestions, ont contribué de manière significative à l'amélioration de ce travail. Bien entendu, les auteurs demeurent seuls responsables des erreurs éventuelles. 


\section{Résumé}

Cet article propose un cadre d'analyse microéconomique de la dynamique territoriale à travers la compréhension des processus de coordination des acteurs locaux. Autant l'approche néo-institutionnaliste que la sociologie économique ont rétabli la place des structures sociales comme ressource et contrainte pour l'action individuelle. Mais, c'est l'entrée par la formation stratégique des liens proposée par l'économie des réseaux qui permet d'appréhender simultanément les dilemmes sociaux de l'action collective, l'évolution des structures globales des relations et le choix décentralisé des actions. Les exigences de la mise en cuvre empirique d'un tel cadre constituent sa principale limite. Son application au contexte spécifique de l'action publique territoriale dans les espaces ruraux, telle qu'elle est présentée dans cet article, permet de renouveler les questions de recherche, quant à la relation entre dispositifs formels - type Parc naturel régional- et réseaux d'acteurs locaux.

\section{Summary}

This paper provides a microeconomic framework of territorial dynamics through the comprehension of the processes of coordination between local actors. Neo-institutional economics as well as economic sociology approaches restore the role of social structures as constraint and resource for individual action. By dealing specifically with the strategic formation of social links, network economics allows to bear simultaneously social dilemmas in collective action, global social structure and individual choices of action. The empirical implementation of such a framework is demanding, and thus constitutes its main limit. However, its application to the specific context of public territorial action in rural areas, as addressed in this paper, confirms its attractiveness for the renewal of research questions in territorial governance, as for the relation between formal devices-like regional natural park in France- and local actor networks.

\section{-1 - \\ Introduction}

Ces trente dernières années, la compréhension du moteur du développement local a été au cœur de la recherche en science régionale. Nous avons ainsi bénéficié de l'éclairage d'approches diversifiées qui ont fait l'objet d'une abondante littérature au travers de la théorie des milieux innovateurs, des districts industriels, de la théorie des ressources spécifiques, etc. Pour autant, il manque encore actuellement un cadre unifié des éléments explicatifs de la diversité des trajectoires de développement local. Un consensus semble néanmoins émerger au sein de ces différentes théories, où ce qui fait finalement la localité du développement est à rechercher dans ce qui est spécifique au territoire étudié, cumulable et capitalisable collectivement. La compréhension du processus de développement au travers de la coordination d'acteurs tient alors une place de choix. Le développement y est avant tout considéré comme la capacité des acteurs situés sur un territoire à en maîtriser l'évolution, à partir d'une vision partagée de la trajectoire que celui-ci doit prendre et qui soit impulsé par une dynamique collective. Une telle approche de la trajectoire des territoires situe ainsi la dynamique de la coordination des acteurs au centre de l'analyse, au sens où elle renvoie à " une capacité créatrice du tissu territorial qui, en organisant une souplesse de combinaison de ses ressources et actifs, dispose de moyens pour mettre en cuvre des stratégies collectives susceptibles d'infléchir le devenir économique du territoire " (COLLETIS et al., 2001). 
Author-produced version of the article publishedithristophe BOSCHET, Tina RAMBONILAZA Revue d'Economie Régionale et Urbaine RERU, 2010, n³, p. 569-593

The original publication is available at http://reru.gretha.u-bordeaux4.fr/

doi : 10.3917/reru.103.0569

Cette dimension relationnelle du développement local a été abondée également par les approches en termes de capital social. Beaucoup de travaux se sont consacrés au rôle du capital social sur le développement économique en dépit des difficultés de convergence de définition, et des problèmes empiriques associés à sa mesure (PONTHIEUX, 2006). La multiplication des travaux sur le capital social n'a pas facilité la compréhension de la notion, d'autant que les sources disciplinaires en viennent à se confondre dans les propositions (LALLEMENT, 2006). DURLAUF et FAFCHAMPS (2005) proposent ainsi une synthèse de la notion de capital social autour de trois grandes idées : i) le capital social génèrerait des externalités positives pour les membres d'un groupe, ii) l'existence de ces externalités est permise par la confiance, les normes et valeurs partagées qui agissent sur les anticipations des agents et leur comportement, iii) confiance, normes et valeurs partagées proviennent d'organisations informelles. L'intérêt accordé aux ressources sociales en tant que dotations individuelles et/ou collectives trouve sa justification à travers les relations qui permettent d'y accéder. Partant, ces dernières améliorent l'efficacité des actions individuelles et collectives par une meilleure circulation des informations et les effets d'autres externalités de proximité. DURLAUF et FAFCHAMPS (2005) insistent alors sur la distinction à faire entre effets de groupe et capital social. En effet, l'existence de relations ne signifie pas systématiquement un réseau, que nous entendons comme des relations structurées auxquelles sont associées des ressources sociales utiles pour l'action, et qui constitue un lieu de coordination des décisions et des actions des individus.

En dehors des problèmes de mesure, l'analyse économique de la dimension relationnelle voire sociologique du développement local au travers du concept de capital social montre les limites d'une approche mésoéconomique de cette question. Une interprétation des ressources sociales en termes de capital, une dotation à un instant donné, ne peut être que très restrictive car non " historicisée " (BARTHELEMY et al., 2004). En outre, on méconnait les mécanismes qui sous-tendent la formation de ce capital social. Néanmoins, s'il est avéré que cette notion permet d'insister sur les effets positifs sur le développement, ou sur les performances économiques des structures sociales du fait d'un mécanisme de coordination particulier, la recherche doit se focaliser directement sur ces structures (DASGUPTA, 2003). Ce sont ces structures sociales qui peuvent faire l'objet de l'intervention de politiques publiques (DASGUPTA op.cit.; DURLAUF et FAFCHAMPS, 2005). Cette approche de l'analyse de la dynamique territoriale, en tant que processus endogène s'articulant autour d'un système d'acteurs auto-organisé, nécessite par conséquent de s'appuyer sur des approches microéconomiques. Plusieurs cadres analytiques peuvent être mobilisés, les éléments constitutifs de ceux-ci sont relayés notamment dans les trois contributions très récentes suivantes : ANGEON (2008), COLLETIS-WAHL (2008), CALLOIS (2007).

Par ailleurs, à plusieurs reprises, l'approche de la coordination des acteurs en termes de réseaux sociaux a été avancée de manière intuitive, comme un cadre analytique pour comprendre la construction, le fonctionnement et l'évolution des territoires. Ce cadre se présenterait comme un outil théorique et empirique unifié de la dynamique territoriale (PECQUeur et ZimMERMANn, 2004 ; Gilly et WAlLET, 2005 ; TernauX, 2007). Pour autant, dans le cas français, les travaux qui mobilisent cette 


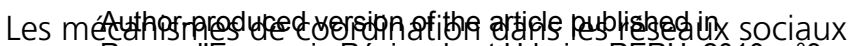

Revue d'Economie Régionale et Urbaine RERU, 2010, n³, p. 569-593

The original publication is available at http://reru.gretha.u-bordeaux4.fr/

doi : $10.3917 /$ reru.103.0569

approche pour appréhender la coordination territoriale restent encore rares, ou sont encore au stade de projet.

Cet article propose de restituer les fondements économiques et les enseignements d'une approche en termes de réseaux sociaux, pour appréhender la formation et la dynamique de coordination des systèmes d'acteurs locaux du développement local. Afin de mieux circonscrire l'intérêt d'un tel cadre et les questions de recherches y afférant, nous nous limitons à titre d'illustration à la problématique de l'action publique territoriale dans les territoires ruraux. Dans ces territoires, les acteurs institutionnels constituent souvent le point de départ des nouveaux projets de développement. Dans cette mesure, le rôle avéré des acteurs publics locaux n'exclut pas la place jouée par les dispositifs institutionnels de l'action publique territoriale, de type parcs naturels régionaux, dans la coordination des acteurs locaux. On est donc amené à s'interroger sur les véritables fondements économiques d'une approche de la dynamique territoriale dans les espaces ruraux, au travers de l'analyse des facteurs explicatifs de la coordination des acteurs locaux.

L'approche en termes de réseaux sociaux de la coordination territoriale impliquant les acteurs publics locaux n'est pas éloignée d'une abondante littérature sur les réseaux de politiques publiques en sciences politiques. Le réseau de politique publique y est défini comme "le résultat de la coopération plus ou moins stable, non hiérarchique, entre des organisations qui se connaissent et se reconnaissent, négocient, échangent des ressources et peuvent partager des normes et des intérêts » (LE GALES et THATCHER, 1995). L'approche usuelle des réseaux des politiques publiques s'intéresse aux configurations de relations entre des acteurs différenciés selon leur statut institutionnel, afin d'analyser le processus de formulation, d'émergence ou de mise en œuvre des politiques publiques. Au sein de cette littérature sur les réseaux de politiques publiques, l'action publique territoriale n'a pas fait l'objet d'une véritable investigation. La seule tentative menée par COLE et JOHN (1995) sur les réseaux locaux de politique publique en France et en Angleterre, les conduit à un certain scepticisme. La notion de relation, centrale dans l'approche, est difficilement traduisible lorsqu'elle concerne des organisations institutionnelles (KENIS et RAAB, 2003). Ces organisations sont liées entre elles par leurs interdépendances en matière de ressources (compétences, autorité, budget etc...), et ne permettent pas lors de l'analyse de souligner le rôle véritable des acteurs au sein d'un système de gouvernance non hiérarchisé. L'approche devient alors un simple outil d'identification des acteurs, au lieu d'un véritable outil explicatif des politiques publiques observées au travers de configurations relationnelles.

Au sein de la discipline, l'évolution du questionnement en matière d'action publique en général, et pour l'action publique territoriale en particulier, invite à réinvestir les dimensions qui structurent les interactions entre acteurs (HASSENTEUFEL, 2008). Cette orientation, d'inspiration néo-institutionnaliste, implique plusieurs challenges : - de mettre en perspective les préférences et les stratégies des acteurs avec leurs rôles ; - d'investir la sociologie des acteurs politiques, comme moyen de production et de diffusion des représentations sociales, délimitant à travers valeurs et théories de l'action les frontières de chaque espace politique (aspect déjà soulevé par BARAIZE et al., 2000); - et enfin de tenter de comprendre la dimension sociale 
Author-produced version of the article published ihristophe BOSCHET, Tina RAMBONILAZA

Revue d'Economie Régionale et Urbaine RERU, 2010, n³, p. 569-593

The original publication is available at http://reru.gretha.u-bordeaux4.fr/

doi : 10.3917/reru.103.0569

des comportements des acteurs afin de mieux expliciter les conflits, les compromis et les contradictions. Le développement d'une approche micro-économique en termes de réseaux sociaux de cette coordination territoriale permet ainsi de fournir quelques éléments de repères à l'approche interactionniste de l'action publique locale en sciences politiques, notamment pour les tenants de la nécessité de postuler la rationalité des choix des acteurs pour l'analyse de l'action publique (DowDING, 2001).

Nous revenons dans un premier temps sur une lecture économique de l'imbrication des coordinations économiques dans les structures sociales. On s'appuie sur une approche néo-institutionnaliste de la coordination, avant de souligner les difficultés méthodologiques que la reconnaissance de cette interrelation soulève (2). La section 3 revient sur quelques points de repères de l'approche en termes de réseaux sociaux des phénomènes économiques, pour déboucher sur l'analyse plus spécifique de la dynamique des réseaux basée sur la formation stratégique des liens entre acteurs. La quatrième section rapporte les enseignements d'une approche empirique de la coordination d'acteurs institutionnels autour d'un projet territorialisé. La section suivante concerne la discussion sur la place des dispositifs institutionnels territoriaux français type "parc naturel régional " pour la coordination locale dans les territoires ruraux. La dernière section conclut et esquisse quelques questions de recherches auxquelles l'approche en termes de réseaux sociaux permettrait de répondre dans le contexte spécifique de l'action publique territoriale.

\section{- 2 - \\ Structures sociales et coordination économique}

Il est difficile d'obtenir spontanément l'alignement des actions individuelles isolées pour qu'elles s'inscrivent au sein d'un collectif qui partagerait la même vision de l'avenir. La question de la coordination des actions entre des agents décentralisés se trouve depuis fort longtemps au centre des préoccupations en économie, que cette coordination passe par le marché ou en dehors de celui-ci. L'observation de l'importance relative des mécanismes de coordination hors marché conduit d'ailleurs au développement de nombreux travaux de recherche sur cette question et la naissance de nombreux courants théoriques. Ces divers courants théoriques tentent d'expliquer de manière approfondie comment des agents, conscients de leurs interdépendances et de leurs divergences d'intérêts, construisent des dispositifs permettant de rendre cohérentes et efficaces leurs actions individuelles. Il s'agit essentiellement pour eux d'édicter des règles et des normes de comportement, de partager des croyances, d'assigner des responsabilités et des rôles permettant par la suite de faire circuler les informations, de partager les ressources, de prendre les décisions les plus efficaces. Les institutions formelles ou informelles (appelées souvent conventions) tiennent ainsi une place importante dans la coordination des agents économiques. Une des principales problématiques de recherche dans ce domaine concerne la coexistence de ces deux dispositifs ou de leurs interrelations, le cœur du programme de recherche de l'approche néo-institutionnaliste. 


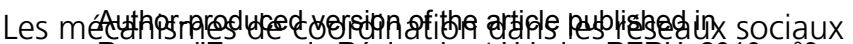

Revue d'Economie Régionale et Urbaine RERU, 2010, n⿳3, p. 569-593

The original publication is available at http://reru.gretha.u-bordeaux4.fr/

doi : $10.3917 /$ reru.103.0569

\subsection{Complémentarité entre relations sociales et dispositif formel de coordination}

L'approche néo-institutionnaliste met l'accent sur le rôle des institutions et dispositifs d'accompagnement ( micro-institutions») permettant de faciliter les transactions et réduire leurs coûts (MENARD, 2003), au sein d'un cadre institutionnel et juridique donné. La solution contractuelle est donc avancée comme forme alternative (au marché ou à la hiérarchie) de coordination. En insistant sur l'importance des propriétés des transactions, la vision williamsonnienne stipule que les institutions encadrent et édictent les interactions entre les individus et déterminent les formes de coordination de leurs échanges (WILLIAMSON, 1985). Cependant, la forme d'interaction est systématiquement envisagée dans un cadre bilatéral. Ce cadre ne permet pas d'appréhender le processus d'émergence des différentes formes de coordination comme un processus d'auto-sélection entre les acteurs, notamment lorsque la solution contractuelle est choisie. Il laisse, en effet, de côté les propriétés des acteurs impliqués qui ici sont a-socialisés. Ainsi, NORTH (1990) et BROUSSEAU $(2000,2001)$ insistent sur la complémentarité entre contrat formel et les ressources sociales comme mode de gouvernance de la coordination d'acteurs. Ils s'appuient sur l'hypothèse selon laquelle, la confiance et les normes de comportements sont générées et entretenues par l'existence d'un cadre légal et juridique qui fonctionne correctement. La faiblesse du cadre légal et juridique favorise ou renforce le recours aux relations sociales qui sont garantes de valeurs, de règles et de normes de comportements partagées par une communauté. Ou, encore, c'est parce qu'en situation d'incertitude et de rationalité limitée des agents, les contrats restant incomplets et les recours juridiques imparfaits et coûteux en cas de défaillance de son partenaire, qu'il devient nécessaire d'encastrer les transactions contractuelles au sein des relations sociales (BROUSSEAU, 2001). Les règles et les normes qui sont générées de manière décentralisée s'érigent ainsi comme des institutions intermédiaires entre l'environnement institutionnel et juridique global et les échanges interindividuels. De ce fait, pour comprendre les logiques de coordination économique à l'œuvre, l'on est amené à reconnaître que les choix économiques incluent une dimension sociale importante. La coordination et sa dynamique sont alors principalement expliquées par les interactions et les réseaux de relations dans lesquelles elles s'inscrivent et relèvent d'un processus d'apprentissage individuel et collectif.

\subsection{Les mécanismes sociaux de la coordination collective}

L'approche néo-institutionnaliste permet de comprendre l'encastrement des transactions économiques ${ }^{1}$ dans les relations sociales, à partir de l'environnement au sein duquel les agents économiques opèrent, et des mécanismes au travers lesquels les relations sociales trouvent leur utilité pour ces transactions économiques. En revanche, elle ne rend pas compte de l'émergence de ces règles et de ces normes. La théorie des systèmes d'acteurs auto-organisés constitue une vision complémentaire en traitant cet aspect, alimentée notamment par la théorie de l'action collective d'Ostrom (1990) qu'elle partage avec une vaste littérature en sociologie (OLSON, 1965 ; OlIVER, 1980 ; MARWELl et OlIVER, 1993 etc.). Cette théorie offre un cadre d'interprétation de l'émergence des règles et des normes au sein d'un collectif 
Author-produced version of the article published ihristophe BOSCHET, Tina RAMBONILAZA Revue d'Economie Régionale et Urbaine RERU, 2010, n³, p. 569-593

The original publication is available at http://reru.gretha.u-bordeaux4.fr/

doi : 10.3917/reru.103.0569

d'acteurs à l'issue d'un processus d'apprentissage à la fois individuel et collectif dans un contexte d'interdépendances stratégiques. Par prolongement, la construction du système de règles communes est devenu l'objet de l'action collective elle-même (OsTrOM, 1992, 1998). La théorie de l'auto-organisation aboutit ainsi sur un cadre explicatif unifié des diversités des solutions de coordination adoptées par les collectifs d'individus selon le contexte socio-économique au sein duquel ils opèrent, et par voie de conséquence selon les dilemmes sociaux auxquels ils font face.

La notion de dilemmes sociaux est empruntée ici à l'approche en termes de jeux $\mathrm{d}^{\prime}$ interactions entre les individus. Ils renvoient à des situations d'opposition entre les structures des intérêts individuels et le bénéfice de l'action collective. En référence à HECKATHORN (1996), on en dénombre 4 types : celui lié à la défaillance du partenaire (dilemme du prisonnier), celui du jeu de dupe (chicken game), celui présentant le conflit entre la sécurité et la coopération sociale (jeu de coordination ou encore jeu de l'assurance), les jeux impliquant l'altruisme. Dans ces contextes, plusieurs variables sont alors évoquées comme influençant l'efficacité relative des règles et des normes collectives : la nature des enjeux, la taille du collectif, l'hétérogénéité des membres, leur expérience passée ou l'historique de leur coordination. Elles impactent le rôle des liens interindividuels qui véhiculent différentes formes de contrôle social sur les décisions individuelles : l'influence, le comportement, l'opinion ou les attentes des membres de la communauté ayant une importance ou faisant partie du voisinage direct de l'individu. Il apparaît ainsi que la lecture même des règles et des normes formalisées doit être mise en perspective par rapport aux principes simples de l'influence des normes sociales favorisant la coopération entre les individus. Ces normes sociales sont : la réciprocité, la confiance, la conscience d'une identité collective ou le leadership (Ostrom, 2000).

L'analyse économique de la coordination collective reconnaît volontiers que dans la réalité, les actions des individus sont influencées par les structures sociales dans lesquelles ils évoluent et que de façon réciproque les individus peuvent agir sur ces structures. Cependant, le sens de cette relation de causalité entre la problématique adressée par les acteurs et le contexte sociologique de leurs actions n'est pas évident. Sont-ce les relations qui ont affecté les choix des acteurs, ou alors les choix qu'ils ont effectué qui ont influencé leur positionnement par rapport à d'autres acteurs? Il est difficile de trouver le meilleur angle d'attaque dès le commencement de l'analyse : s'intéresser aux actions individuelles et à leurs impacts sur les structures sociales (souvent la posture adoptée du point de vue théorique en économie), ou privilégier dès le départ la compréhension des mécanismes collectifs qui assurent la cohésion de ces actions individuelles (une approche plus sociologique et empirique).

Ces dernières années, l'on assiste à un foisonnement de la littérature mobilisant une analyse en termes de réseaux sociaux des phénomènes économiques faisant intervenir des interactions entre les individus, en accordant une place importante aux propriétés des structures sociales qui soutiennent ces phénomènes. Cette approche ne se limite pas seulement à la livraison d'éléments de compréhension d'un état de coordination donné, au travers l'identification des ressources sociales partagées au sein du collectif d'acteurs. Elle rend compte des mécanismes par lesquels les 


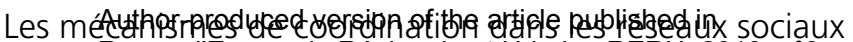

Revue d'Economie Régionale et Urbaine RERU, 2010, n`3, p. 569-593

The original publication is available at http://reru.gretha.u-bordeaux4.fr/

doi : $10.3917 /$ reru.103.0569

ressources sociales (normes et règles de comportement) sont générées, produites, circulent et organisent elles-mêmes la nouvelle configuration des relations entre les individus. L'approche en termes de réseaux sociaux de la coordination permettrait de lever certaines limites de l'approche économique traditionnelle de la coordination collective, à la fois du point de vue théorique mais aussi empirique. Elle est d'ailleurs avancée comme un outil complet pour appréhender et formaliser l'émergence et la dynamique de différentes formes de gouvernance de la coordination d'acteurs (COHENDET et al., 2003). L'objectif de la section suivante consiste alors à revenir sur le contenu d'une approche en termes de réseaux sociaux permettant de traiter avec plus d'acuité la coordination territoriale.

\section{- 3 - \\ Comportement(s) individuel(s) et systèmes de relations sociales : enseignements de la littérature}

\subsection{Fondements de l'approche structurale en sociologie}

Deux idées attribuées à GRANOVETTER ont contribué au rapprochement entre disciplines sous la bannière de l'étude de l'objet « réseau ». La première envisage l'encastrement des phénomènes économiques dans les relations sociales (GRANOVETTER, 1985). Il s'agit de considérer que les relations économiques entre individus ou organisations s'appuient sur un ensemble préexistant de relations sociales, en réponse critique à une vision sous-socialisée des phénomènes économiques que la figure du marché rendait compte. Il en découle deux propositions fondamentales (GRANOVETTER, 2000) : i) l'action est toujours socialement située et ne peut être expliquée seulement par les motifs individuels; ii) les institutions sociales ne sont pas automatiquement produites sous une forme incontournable, mais elles sont socialement construites (p. 95-96).

La seconde idée, en spécifiant la diversité des contenus et l'intensité variable des liens qui véhiculent la relation économique, permet de distinguer les liens forts des liens faibles (GRANOVETTER, 1973), et avancer la notion de structure. Les liens forts caractérisent des contacts fréquents, un partage de " ressources ", l'échange de services réciproques etc., tandis que les liens faibles se distinguent par des contacts peu fréquents et peu intenses en termes de contenu. D'un point de vue économique, chaque type de lien présente un avantage : les liens forts assurent une permanence d'échange entre les agents, mais les liens faibles permettent une diversité dans les services et ressources fournis, puisque l'un et l'autre des agents connectés dépendent de réseaux sociaux différents (GRANOVETTER, 1985).

Les travaux qui ont découlé de la théorie de GRANOVETTER ont donné lieu à un essor qui s'est traduit par une formalisation des concepts en accordant une place de plus en plus importante à la structure des relations. Le fait que certains individus tirent avantage de leurs relations sociales est à relier avec leur positionnement dans cette structure et la configuration des liens qu'ils entretiennent avec d'autres (BURT, 
Author-produced version of the article published ihristophe BOSCHET, Tina RAMBONILAZA Revue d'Economie Régionale et Urbaine RERU, 2010, n³, p. 569-593

The original publication is available at http://reru.gretha.u-bordeaux4.fr/

doi : 10.3917/reru.103.0569

1992). En d'autres termes, "la forme du réseau a une incidence sur les ressources qu'un individu peut mobiliser et sur les contraintes auxquelles il est soumis. Elle ne le détermine pas, mais elle explique que tout ne soit pas possible pour lui et que dès lors certains comportements ou stratégies soient, en raison de la position occupée dans le réseau, plus probables que d'autres » (FORSÉ, 2008, p. 11). La mise en évidence de l'importance de la structure des relations pour la compréhension des actions individuelles a connu un essor important jusqu'à la construction d'une méthodologie particulière, l'analyse des réseaux sociaux, depuis l'ouvrage de WASSERMAN et FAUST (1994). Dans ce cadre, la formalisation se concentre sur la caractérisation des liens qui composent un réseau de relations (approche structurale) au détriment de la caractérisation des relations entre individus (approche structurelle). Il en va ainsi pour l'histoire et les modalités de constitution des liens formant le réseau. Cette conception permet d'appréhender le réseau quelle que soit la nature des nœuds (individus, organisations, secteurs...) et la nature des liens. Il s'agit d'abord de mettre en évidence des éléments significatifs de la structure du réseau (comme par exemple des sous-ensembles - cliques - ou par le biais d'indices statistiques : densité, connectivité, équivalence...).

La théorie des "trous structuraux" de BuRT ${ }^{2}$ peut de cette manière passer comme une interprétation formelle de la théorie des liens faibles de GRANOVETTER (1973). Chez BURT, ce n'est pas la force du lien qui conditionne la pertinence de l'information véhiculée, mais la caractéristique structurale du réseau au sein duquel cette information est transmise, en l'occurrence la place occupée par l'individu qui l'émet. D'où l'importance des trous structuraux qui soutiennent la présence d'un individu constituant un " pont » entre deux ensembles de relations dont celui-ci est susceptible de tirer profit. On considère par là que le " trou » formé par l'absence de liens entre différents groupes d'individus implique la circulation d'informations différentes dans chacun d'eux. Pris dans leur ensemble les deux flux d'information sont non redondants. Selon le contexte, la position d'intermédiaire occupée par l'individu qui établit le lien entre les deux groupes lui procure des avantages selon la stratégie qu'il adopte, et les bénéfices qu'il compte retirer de cette position : le pouvoir, le contrôle et le prestige associés à la diffusion d'informations non redondantes. Autour de cette théorie, BuRT met ainsi en balance deux configurations de liens aux propriétés distinctes. Les réseaux de relations riches en trous structuraux procurent les avantages décrits précédemment aux individus qui occupent ces positions, et sur le plan collectif ces avantages se traduisent par la possibilité d'être connectés à des acteurs distants via les intermédiaires. D'un autre côté, les réseaux de relations denses où tous les individus sont interconnectés facilitent la circulation rapide et la fiabilité de l'information. Les propriétés de ces configurations ont des conséquences opposées sur la coopération, en partant de l'idée que la structure du réseau est révélatrice du degré d'interconnaissance des individus, selon un processus cumulatif étiré dans le temps. D'une manière que l'on pourrait considérer similaire aux modèles de réputation de KREPS (1990), les agents prennent des informations utiles pour l'action au sein du groupe. Cette possibilité est aisément concevable dans les réseaux denses, beaucoup moins dans les réseaux présentant des configurations éparses. BURT rassemble ce phénomène de contrôle social sous la bannière de la confiance, qui contribue à faciliter l'anticipation des coopérations et diminue ainsi les risques qui y sont associés (COLEMAN, 1988, 1990). Réciproquement, dans les réseaux de relations 


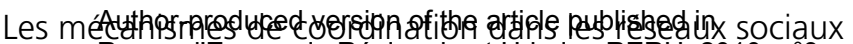

Revue d'Economie Régionale et Urbaine RERU, 2010, n`3, p. 569-593

The original publication is available at http://reru.gretha.u-bordeaux4.fr/

doi : $10.3917 /$ reru.103.0569

plus lâches, riches en trous structuraux, la construction de relations comporterait une part de risque dans la mesure où le mécanisme de la confiance ne joue plus.

On comprend intuitivement l'intérêt de ces énoncés à un niveau d'observation macro des structures. La coordination des acteurs serait basée sur un équilibre entre la qualité des relations reposant sur une confiance socialisée vecteur de coopération interindividuelle (Closure), et la recherche de ressources utiles pour l'action via des relations distantes et non redondantes (Brokerage). Sur un plan collectif, les avantages procurés par les deux types de structures renforcent leur co-existence. La présence de " trous » alimente le réseau dense en ressources nouvelles, et la présence de réseaux denses procure un avantage stratégique à la position d'intermédiaire.

Des limites sont néanmoins à souligner. L'analyse est tout d'abord livrée en statique. La théorie de BURT ne nous apporte pas d'enseignement sur le processus de formation et de co-existence de ces configurations. Ce qui nous amène à la critique principale, qui soulève la nécessité d'un cadre micro de l'approche. Bien qu'implicitement avancé comme un agent stratège via la recherche d'une position avantageuse, BURT n'avance pas de « rational » derrière le comportement de l'individu, si ce n'est qu'il précise un degré d'hétérogénéité supérieur au niveau intragroupe qu'intergroupe (BURT, 2004). Si occuper un trou structural procure un avantage compétitif pour un individu, on peut alors s'attendre à ce que tous les individus soient à la recherche de cette position. Or si c'était le cas, il n'y aurait à l'issue du processus aucun agent occupant ce trou structural. On peut donc penser que lorsque ces positions sont occupées c'est qu'elles sont le fait du consentement des autres. Un éclairage sur les configurations relationnelles recherchées par les acteurs apparaît alors nécessaire, et notamment par la dimension stratégique de la formation des liens proposée par le versant économique de l'approche des réseaux.

\subsection{L'analyse économique de la formation stratégique des liens}

L'article séminal de JACKSON et WOLINSKY (1996) a fourni les fondements théoriques de la formation stratégique des liens comme base de l'analyse de la formation et de la dynamique des réseaux d'acteurs. La formalisation mobilise la rationalité de l'intérêt personnel des agents pouvant créer, maintenir ou détruire des liens. Le choix des liens est opéré selon un calcul coûts-bénéfices inhérents à la structure du réseau et donc de la réaction des autres agents lors de la formation des liens. Cette prise en compte d'externalités de proximité au sein d'un réseau de relations permet de comprendre comment se forme la coordination à travers l'interprétation que se font les agents de leur environnement stratégique. La forme du jeu qui gouverne l'évolution des liens est donc non-coopérative. Il est remarquable de constater cependant, qu'à l'instar d'autres contributions qui s'inscrivent dans cette démarche (et parmi eux DutTA et al., 1998 ; BALA et GOYAL, 2000), il n'existe pas de forme explicite du jeu entre les joueurs.

Au sein de ce processus de formation endogène du réseau, l'état final du réseau est défini sous l'angle de deux critères : i) dans la mesure où l'utilité de chaque agent dépend du réseau, la recherche de stabilité des stratégies permet d'en déduire les 
Author-produced version of the article published ihristophe BOSCHET, Tina RAMBONILAZA Revue d'Economie Régionale et Urbaine RERU, 2010, n³, p. 569-593

The original publication is available at http://reru.gretha.u-bordeaux4.fr/

doi : 10.3917/reru.103.0569

propriétés de sa structure. Un réseau est dit stable lorsqu'il n'y a plus de tentation pour les agents à créer ou à détruire des liens, solution établie au travers du critère de NASH. ii) Sur un plan macro, la caractérisation du réseau s'élabore sur un critère donné d'efficacité renvoyant au bien-être social. Un réseau est dit efficace quand sa valeur est supérieure à toute autre forme de réseau possible. Au travers de ces critères de stabilité et d'efficacité se manifeste la relation entre l'alignement des incitations individuelles et le bien-être collectif, que l'on retrouve le plus souvent sous forme de dilemmes sociaux (DEROIAN, 2003). Tous les réseaux ne sont pas stables et efficaces, résultat d'une tension entre l'intérêt stratégique individuel et l'intérêt collectif. Ce qui nécessite de connaître la fonction d'allocation des rétributions entre les membres du réseau, pour savoir si le montant de la rétribution reçu par chacun est supérieur ou égal à celui qu'ils auraient perçu dans d'autres réseaux.

En plus de la décision de formation des liens, des contributions récentes incorporent la situation dans laquelle chaque agent doit adopter un mode de comportement face à ses partenaires (GOYAL et VEGA-REDONDO, 2000 ; JACKSON et WATTS, 2002). Ces recherches se focalisent sur les incitations des agents à choisir l'action la plus efficace, l'objectif étant de connaître la nature de la relation entre la structure du réseau et le choix de l'action. Plus précisément, il s'agit d'appréhender de façon simultanée le choix du lien et de l'action qu'il sous-tend : les agents peuvent se coordonner sur une action représentant un optimum social et individuel, ou sur une action efficace en termes de gains mais où l'on ne peut écarter un risque de défection de la part de certains participants par la suite. Ce cadre permet à GoYAl et VEGAREDONDO d'avancer un résultat majeur. L'arbitrage individuel entre coûts et bénéfices donne lieu à un ensemble restreint de configurations stables qui correspondent soit à une configuration complètement connectée, soit à une configuration en étoile. Ce résultat permet aux auteurs de mettre en évidence deux processus. À coût de formation de lien moindre, l'attraction relative des actions alternatives influe peu et les agents se connectent quelle que soit l'issue de l'interaction, et alors la structure de réseau émergente est complète (à condition de bénéfices garantis). En revanche, le processus de formation du réseau influe sur le comportement de choix d'action des partenaires : à coût élevé de formation des liens, les agents se coordonnent sur une action efficace, au cas contraire sur une action non garantie contre le risque de défaut.

Le point de départ de l'approche économique insiste sur le caractère stratégique de la formation des liens où sont pris en compte les choix des autres par des externalités de proximité. Des approfondissements nous livrent, par la suite, au travers d'une véritable interaction stratégique, la façon dont les agents se coordonnent. La mise en évidence de l'influence de la structure sur le type d'action qu'ils sélectionnent permet d'introduire la problématique de la défection dans la coordination, la configuration des réseaux étant elle-même définie par la formulation des gains nets des agents.

Tant du côté de l'approche sociologique qu'économique des réseaux, peu d'éléments permettent d'abonder précisément sur les structures relationnelles recherchées par les individus. Du côté de la sociologie, BuRT avance explicitement la confiance 


\section{Les mécuthpsraresdureduersiph \&fithr artiple publishedanx sociaux}

Revue d'Economie Régionale et Urbaine RERU, 2010, n`3, p. 569-593

The original publication is available at http://reru.gretha.u-bordeaux4.fr/

doi : $10.3917 /$ reru.103.0569

comme mécanisme de coordination, sous-tendue par l'articulation de deux configurations structurales, les réseaux denses et les réseaux riches en trous structuraux. Si d'un point de vue micro, ces deux configurations constituent des extrêmes typiques, on peut considérer sans difficulté que le raisonnement doit être affiné : en admettant une hétérogénéité parmi les membres d'un réseau, la diversité des stratégies invite à reconnaître des architectures intermédiaires de réseaux. Le versant économique quant à lui contribue d'une façon quasi-exclusivement théorique à la question car du fait de la difficulté de disposer de base de données relationnelles fournie, la plupart des tests empiriques se cantonne à la simulation numérique (voir BURGER et BUSKENS, 2009 ; FALK et KOSFELD, 2003 ; et KOSFELD, 2004) pour une revue de la littérature sur le sujet).

\section{-4 - \\ La problématique de la stratégie d'investigation empirique}

Le versant empirique des analyses des réseaux s'est aujourd'hui focalisé sur leur dynamique. La nature des relations, la composition de la structure et les propriétés qui en découlent évoluent selon des mécanismes micro qui influent sur la structure globale. La question de la dynamique renvoie à plusieurs dimensions du travail de recherche. Il s'agit tant des outils statistiques susceptibles de rendre compte au mieux de ce processus, que du type d'analyse (intérêts d'une approche longitudinale versus en coupe), et enfin de la stratégie de récoltes de données. On voit ensuite, à partir d'une étude empirique révélatrice, que cette dernière dimension soulève d'ailleurs la question de la manière d'investir empiriquement la stabilité et la permanence des liens.

\subsection{L'exploitation des données relationnelles en question}

L'analyse empirique des données relationnelles pour caractériser les réseaux sociaux a fait l'objet d'une panoplie de méthodes. Une catégorie d'analyses permet de caractériser la structure globale d'un réseau d'une manière relativement simple au travers d'indicateurs synthétiques (comme la densité, la centralité ou encore l'identification de sous-ensembles cohésifs, à ce sujet voir WASSERMAN et FAUST, 1994). Une autre catégorie d'analyses se positionne sur le développement de modèles statistiques de réseau. Plusieurs arguments plaident en faveur de cette démarche, dont le principal est de pouvoir relier les influences réciproques entre les niveaux micro (les dyades) et macro (structure globale) d'un réseau (Robins et al., 2007).

L'histoire du développement des modèles statistiques des réseaux peut être résumée par la poursuite de l'objectif suivant : mettre en évidence les déterminants de la formation et l'évolution d'un lien entre deux individus, eux-mêmes imbriqués dans une structure plus globale, faisant intervenir d'autres acteurs et dont les propriétés évoluent avec les liens entre ceux-ci. Chaque individu membre du réseau constitue l'unité d'observation de l'existence ou non des liens avec les autres membres. La 
Author-produced version of the article published ihristophe BOSCHET, Tina RAMBONILAZA Revue d'Economie Régionale et Urbaine RERU, 2010, n³, p. 569-593

The original publication is available at http://reru.gretha.u-bordeaux4.fr/

doi : 10.3917/reru.103.0569

formation des liens observés peut relever d'un processus entièrement stochastique ou être en partie influencée par un ensemble de variables exogènes comme les attributs des paires (e.g., les variables « sexe » et « âge » dans les réseaux d'amitié). Les modèles statistiques s'appuient par conséquent sur une formalisation probabiliste de la formation des liens pour caractériser les propriétés structurales ${ }^{3}$ des réseaux (centralité, densité, ensemble cohésifs...).

La difficulté pour la modélisation est que l'on ne peut faire l'hypothèse que ce lien est observé indépendamment des autres liens ${ }^{4}$. Du fait de cette dépendance statistique, les modèles doivent rendre compte de l'émergence de la structure du réseau notamment (mais pas seulement) à partir d'effets se produisant à des niveaux différents de l'architecture des liens. La classe des modèles ERGM (Exponential Random Graph Models, voir RoBIns et al., 1999 ; WASSERMAN et PATISSON, 1996) considère alors que l'ensemble des liens traités sont corrélés entre eux. A partir des liens observés, il s'agit de partir d'une décomposition du réseau en la plus petite souscomposition, la triade, et ses 16 configurations possibles, selon la nomenclature de classes isomorphiques fournies par HOLLAND ET LEINHARDT (1976) : trois acteurs avec des relations ou non, réciproques ou non. Les modèles ERGM permettent par la suite de simuler à partir des distributions empiriques de ces sous-structures particulières la probabilité d'émergence de la structure globale du réseau en référence à des propriétés structurales de configuration théorique. De cette manière, en passant par l'analyse de configurations élémentaires, l'estimation paramétrique du modèle de configuration locale des liens permet d'observer quels effets structuraux (la réciprocité à un niveau dyadique, la transitivité à un niveau triadique, la densité à un niveau global) renforcent la probabilité d'occurrence du réseau dans son ensemble. Le modèle n'exclut pas bien évidemment les effets de variables exogènes comme les caractéristiques des acteurs - les attributs d'ego et d'alter- et les caractéristiques de la relation en elle-même - fréquence, similitude - . Ce traitement statistique concerne essentiellement les bases de données en coupe (une seule observation au travers d'une seule enquête). La question de la dynamique du réseau n'est pas traitée directement.

Développées essentiellement en sociométrie, les méthodologies présentées cidessus ne nécessitent donc aucune hypothèse quant à la motivation des individus pour établir ou détruire les liens. Les liens observés sont associés à des processus sociaux génériques (affinité, solidarité, réciprocité, sympathie). L'inscription de cette formalisation de la formation des liens dans une démarche stratégique envisagée du point de vue de chaque ego (l'individu comme unité d'observation des liens) a débouché sur le modèle acteur-orienté, SIENA (Simulation Investigation for Empirical Network Analysis [voir SNIJDERS et al. (2009) pour un aperçu exhaustif récent].

Le modèle repose sur l'hypothèse que l'acteur, orienté par un objectif particulier, effectue des choix stratégiques relativement à la formation, au maintien ou à la suppression de ces liens au sein d'un cadre de maximisation de l'utilité qu'il en retire. L'acteur est donc maître de ses relations, ce qui guide l'évolution ultérieure du réseau. Plus précisément, chaque acteur choisit ses relations afin d'en retirer un avantage individuel et influer sur la structure globale du réseau. Pour refléter au mieux son 


\section{Les mécuthpsraredurecturisiph afitha artiple publishodinx sociaux}

Revue d'Economie Régionale et Urbaine RERU, 2010, n³, p. 569-593

The original publication is available at http://reru.gretha.u-bordeaux4.fr/

doi : $10.3917 /$ reru.103.0569

comportement stratégique, on postule que l'acteur a une connaissance totale de la configuration des liens dans le réseau au moment où il réalise son choix.

L'avantage de ce modèle est de développer une méthodologie d'analyse de la formation et de la dynamique des réseaux au travers des déterminants des relations dyadiques ou triadiques, comme pour le modèle ERGM plus haut, en considérant que les caractéristiques structurales sont endogènes. En d'autres termes, les liens et les autres variables déterminant de ces liens influencent les caractéristiques structurales. L'emboîtement du processus de la formation des liens et du processus d'évolution du réseau permet de traiter de manière effective la dynamique du réseau à partir de la formation des liens. Le modèle SIENA constitue ainsi la forme généralisée des modèles de dynamique de réseau initiés par WASSERMAN (1980) et qui nécessitent de disposer d'au moins deux jeux de données correspondant à des périodes distinctes dans le temps. Ici, une approche en coupe permet d'abonder la dynamique des réseaux, en faisant l'hypothèse que les motivations des individus sont stables dans le temps, et donc, les liens aussi.

Le degré de complexité atteint dans le domaine de l'analyse statistique des réseaux sociaux n'élude pas pour autant l'importance de la récolte de données dans la stratégie d'investigation empirique. En amont, la manière de reconstruire le réseau conditionne les modalités de l'enquête proprement dite. Elle peut s'effectuer de façon complète i.e., les frontières du système sont circonscrites et toutes les relations entre les acteurs sont examinées. Une alternative est de procéder par la construction de réseaux personnels, dans le cas où l'on est face à un réseau ouvert, c'est-à-dire dont les délimitations sont impossibles à observer (FERRAND et DE FEDERICO DE LA RUA, 2004). Pour ces derniers, on procède à un échantillonnage d'acteurs au sein d'une population de référence, et l'enquête consiste à reconstruire l'étoile des relations qu'ils entretiennent, mais aussi à révéler leur perception des relations que leurs alters entretiennent entre eux. Bien que les approches semblent radicalement différentes, les approximations statistiques des sous-structures typiques d'un réseau complet (i.e., les probabilités d'occurrence de configurations relationnelles) semblent se rapprocher des approximations de l'analyse de réseaux personnels (FERRAND, 1997). La pertinence du choix de la stratégie de collecte de données est soumise - en plus de la question des possibilités de délimitation et d'observation du système - aux conséquences des relations indirectes avec l'acteur en question, et donc ses limites cognitives (DE FEDERICO DE LA RUA, 2004). L'étendue pertinente des conséquences des liens peut être plus large que celle cantonnée au réseau centré sur Ego (e.g., les effets de l'occupation d'un trou structural), et peut donc nécessiter la prise en compte d'effets purement structuraux. Dans cette perspective, l'approche par un réseau complet est à privilégier.

Par ailleurs, on se doit également de questionner la pertinence de la technique de récolte de données avec la question qui nous anime (l'action publique locale). A partir du moment où l'observation se fait sur un nombre délimité d'acteurs, il est possible d'obtenir des informations sur les relations entretenues entre chacun à partir de deux sources de données. Elles peuvent être de nature documentaire selon le cas que l'on veut observer (données d'archives, procès verbaux de réunions, signatures 
Author-produced version of the article published ihristophe BOSCHET, Tina RAMBONILAZA Revue d'Economie Régionale et Urbaine RERU, 2010, n³, p. 569-593

The original publication is available at http://reru.gretha.u-bordeaux4.fr/

doi : 10.3917/reru.103.0569

de contrats...). Si l'observation complète par voie documentaire n'est pas possible, il reste l'alternative de l'administration de questionnaires à tous les membres du système observé. On utilise dans ce cas-là un générateur de noms, en soumettant une liste de noms à l'enquêté et en lui demandant de sélectionner ceux avec qui il entretient des relations. Dans le cas d'une récolte de données par voie documentaire, l'information acquise est fiable mais est soumise à la question de son exhaustivité par rapport au système que l'on observe. Dans la situation où le questionnaire est choisi, l'exhaustivité des informations est plus facilement atteinte, et la qualité des données dépend de façon cruciale de la construction du questionnaire en rapport avec la question posée.

Le problème qui se pose alors est la pertinence du choix méthodologique d'observation du réseau (complet versus personnel) et la question du recueil des données. Dans le cas d'une reconstruction complète du réseau, il s'agit de bien faire correspondre l'existence de liens entre deux personnes, au travers du contexte de leur construction. Les enseignements issus de la théorie économique nous apprennent que des déterminants structuraux impactent sur la formation des liens et sur le choix des actions mutuellement adoptées. La coordination sur le choix des actions, tout comme la question de l'influence des déterminants structurels et structuraux sur ce processus, est à notre connaissance passée sous silence dans la littérature sur le traitement statistique des données relationnelles. Une tentative est portée par le développement économétrique autour du traitement des effets de paire [voir Bramoullé et ForTiN (2009) pour une revue détaillée], mais l'introduction de l'influence des propriétés structurales n'est encore qu'au stade de chantier de recherche.

\subsection{Dilemmes sociaux et stratégies de coordination : un exemple d'analyse empirique}

Il s'agit ici de s'intéresser à l'analyse de l'influence de la structure du réseau des relations existantes sur les choix des partenaires par les acteurs. Ce lien de causalité empirique permet, en effet, de repérer les dilemmes sociaux que les acteurs essaient de traiter lorsqu'ils activent leurs liens pour résoudre un problème spécifique. Les acteurs sont bien évidemment stratégiques. Ainsi, à partir des données recueillies auprès des acteurs publics de dix territoires estuariens américains, BERARDO et SCHOLZ (2008) analysent les fondements des choix de partenariats développés par chaque acteur sur leurs territoires respectifs qui nécessitent d'allier à la fois développement économique et préservation de l'environnement. Les réseaux d'acteurs publics observés présentent des caractéristiques particulières puisqu'ils ne font pas l'objet d'une réglementation fédérale particulière et sont ainsi soumis à une problématique de gestion collective de la zone délimitée par les limites physiques de chaque estuaire. En s'attachant aux projets de collaboration effectués entre organisations (194 organisations enquêtés en deux vagues, en 1999 et en 2001) concernées par la mise en œuvre de projets sur ces espaces (sans distinction de leur finalité : économique, environnementale, d'aménagement, etc..), l'hypothèse que les auteurs cherchent à tester est la suivante : en situation de confiance, la recherche d'une transmission d'information efficace serait recherchée, alors qu'en situation de confiance faible, la recherche de transitivité et de réciprocité serait préférée pour limiter les risques de défection. L'analyse 


\section{Les mécuthpsraregduredursiph \&fitha artiple publishedinx sociaux}

Revue d'Economie Régionale et Urbaine RERU, 2010, n`3, p. 569-593

The original publication is available at http://reru.gretha.u-bordeaux4.fr/

doi : $10.3917 /$ reru.103.0569

s'effectue alors par l'appariement des positionnements de chaque partenaire (ego et alter) au sein du réseau. Il est entendu ici que la situation de confiance ou non est définie par des propriétés structurales du réseau, tel que nous l'avons énoncé auparavant et le détaillons ci-après.

Dans un climat de confiance élevée, la recherche d'une transmission efficace de l'information se traduit de deux manières. La recherche de proximité entre acteurs permettrait une plus grande efficacité dans la transmission d'informations. Du point de vue d'ego, cette proximité peut être envisagée de deux manières. Dans un cas, Ego peut envisager une relation avec un alter avec lequel il n'est pas lié ni directement ni indirectement, mais dont la formation de lien présente l'opportunité d'accéder à des informations nouvelles par le biais des liens qu'entretient alter avec d'autres. On retrouve ici les arguments de l'ouverture du réseau vers l'extérieur de BuRT (1992). Il peut dans d'autre cas s'agir d'une recherche de relation plus directe avec un alter, séparée par un nombre d'intermédiaires qu'égo chercherait à réduire. Ici, le renforcement des liens à l'intérieur du réseau renforce la caractéristique communautaire, ou en d'autres termes la tendance à vouloir former des triades.

On peut également aboutir à une efficacité similaire en termes de transmission d'informations à travers un réseau en étoile. La recherche de lien avec un acteur déjà central sera dans ce cas le leitmotiv de la dynamique. Ce type de réseau est supposé efficace du point de vue de la transmission d'information, puisque tous les coûts associés à la tâche sont confiées à une seule personne. Une telle structure si elle perdure dans le temps aurait des effets négatifs sur les capacités d'apprentissage des membres dont les possibilités d'accès à de multiples sources d'informations sont limitées (LEAVIT, 1951). Ce raisonnement n'apparaît donc valable que, toutes choses égales par ailleurs, pour les réseaux de petite taille. Par ailleurs, le calcul et l'emploi de la centralité se décline à travers plusieurs indicateurs. La centralité de proximité mesure l'autonomie d'un acteur (FREEMAN, 1979). Elle traduit le degré d'éloignement d'un individu par rapport à un autre. La centralité d'intermédiarité, mesure la capacité de contrôle et de coordination d'un acteur (Lemieux et Ouimet, 2004). Cette mesure, qu'elle soit effectuée au niveau individuel ou collectif, traduit plusieurs situations : la transmission d'information complexes (due à la coexistence de groupes faiblement connectés), la présomption de l'hétérogénéité des acteurs dans le réseau. WASSERMAN et FAUST (1994) prennent notamment le célèbre exemple des familles florentines de PADGeTt et ANSELL (1993) et la position centrale d'intermédiaire jouée par les MÉDICIS. A contrario, la présence d'acteurs/groupes intermédiaires centraux fragilise la structure des relations et contrarie la production de confiance.

En situation de risque associé à la défection, les résultats attendus en termes d'analyse des choix d'appariement sont les suivants :

- une recherche de réciprocité correspond à un niveau dyadique un meilleur accès à l'information, de la production de confiance et une réduction de l'incertitude sur l'issue des coopérations futures. Comme il est demandé dans l'enquête à chaque individu de demander avec qui il communique au sujet de la problématique 
Author-produced version of the article published Thristophe BOSCHET, Tina RAMBONILAZA

Revue d'Economie Régionale et Urbaine RERU, 2010, n³, p. 569-593

The original publication is available at http://reru.gretha.u-bordeaux4.fr/

doi : 10.3917/reru.103.0569

environnementale de leur territoire, l'identification de liens réciproques est aisément identifiable.

- une recherche de transitivité consiste à densifier ses relations. Il s'agit ici de réduire les coûts associés au contrôle et à l'exécution de sanction. Incidemment, la production de transitivité renforce la crédibilité des engagements. Cette recherche de transitivité est détectable par l'observation de triades au sein du réseau.

Nous ne présentons ici que les principaux résultats de BERARDO et SCHOLZ (2008) quant aux réseaux d'acteurs observés sur les dix estuaires qu'ils investissent. Dans l'analyse en coupe, la recherche de centralité est significative, ce qui confirme un phénomène de sélection favorisant les acteurs déjà centraux dans la structure des relations. Le paramètre associé à la recherche de proximité est positif mais non significatif, à laquelle lui sont préférées la recherche de transitivité et de réciprocité. Les structures de relations associées à une situation de confiance forte sont ainsi préférées. L'analyse des données longitudinales confirme d'une manière générale les résultats de l'analyse en coupe. Ainsi les paramètres associés à la recherche de transitivité et de proximité ne sont pas significatifs. La relation confiance-choix du partenaire dans le réseau est, par contre, plus à mettre en faveur d'un phénomène d'influence, contrairement aux résultats de l'analyse en coupe. D'un point de vue théorique, les auteurs concluent que la recherche de coordination au travers d'un acteur central est le choix dominant. Une source d'explication valable selon les auteurs est mobilisée au travers de l'ancienneté de la coopération. Plus la formation d'un réseau d'acteurs publics est récente, plus la sélection d'un partenaire central est probable. Cette forme de coordination serait privilégiée en raison des gains immédiats qu'elle procure. En revanche BERARDO et SCHOLZ (op. cit., 2008) postulent qu'avec le temps les rentes ainsi dégagées s'épuisent, et la recherche de coopérations supplémentaires implique de cette façon une recherche de réciprocité et de transitivité pour limiter les risques de défection, caractéristiques des réseaux plus anciens car plus ouverts.

En appui à ce qui est avancé au préalable de cette sous-section, les enseignements de l'analyse de BERARDO et SCHOLZ (2008) suscitent tout autant de questions. Les auteurs insistent sur le fait que l'émergence du leader (acteur central) n'est pas le fait d'attributs individuels (compétences, ressources budgétaires...) mais le fait de déterminants structuraux qui sous-tendent le problème de coordination. On peut accepter ce résultat comme un fait avéré ou le considérer comme un artefact, dans la mesure où le réseau analysé est obtenu à partir de liens inférés des contextes des actions plutôt que des actions elles-mêmes. Une autre explication possible, mais qui n'est pas explorée par les auteurs, serait alors à rechercher du côté de la nature des actions sur lesquelles les acteurs sont engagés. A ce stade de la réflexion, la question de la simultanéité du choix de l'action et du choix de partenaire reste ouverte. 


\section{Dispositifs institutionnels territoriaux et mécanismes de coordination}

\subsection{Le PNR comme cadre d'un processus de négociation endogène des acteurs locaux}

Les PNR ont été désignés à l'origine pour impulser, dans des espaces en difficulté mais dotés d'un patrimoine naturel et culturel important, des projets de développement territorial alliant valorisation économique (au départ beaucoup orientée vers le développement du tourisme) et préservation des aménités naturelles et culturelles. Ces projets de développement territorial sont inscrits au sein d'un dispositif contractuel qui engage pour 12 ans les acteurs locaux. Les PNR définissent à la fois le contenu et le périmètre du projet collectif et le mode gouvernance de la coopération entre les acteurs au sein de ce nouveau territoire à travers la charte. Les négociations autour de la construction ou du renouvellement des Parcs Naturels Régionaux (PNR) peuvent ainsi être considérées comme la révélation du souhait des acteurs locaux d'impulser une nouvelle trajectoire pour leur territoire. Le processus de négociation des projets des PNR est souvent assez long. On s'étonne par ailleurs pourquoi, des acteurs qui ont l'habitude de travailler ensemble de par leur proximité géographique ou ayant évolué auparavant dans des projets communs soit par nécessité, soit du fait des règles institutionnelles imposées par l'Etat, mettent du temps à se coordonner lorsqu'il s'agit de mettre en place un dispositif tel que le PNR.

Les spécificités de la charte sont maintenant bien connues. On en relève trois qui nous intéressent plus spécifiquement ici : la notion de partenariat entre ses signataires pour la mise en œuvre des orientations d'action négociées, la souplesse du cadre juridique - voire l'absence de forme juridique préétablie - et l'absence (à quelques rares exceptions près) de modalités de sanctions (et donc de contrainte juridique) garantissant la mise en œuvre des programmes d'actions (BILLET, 2006). La charte constitue ainsi les repères pour l'action collective, c'est-à-dire une liste de règles du jeu (dans une approche northienne). Cependant, si ces repères sont formulés au sein d'un dispositif formel qui autorise une certaine souplesse permettant aux acteurs de s'adapter et de faire émerger de nouvelles dynamiques, l'absence de recours légal n'exclut pas la défaillance des partenaires ${ }^{5}$. Sans abonder une vision manichéenne de la coordination collective, l'existence d'une probabilité forte de défaillance des partenaires renvoie à l'importance des ressources sociales comme complémentaires de ces dispositifs pour soutenir l'action collective. La structure sociale au sein desquels les principaux acteurs évoluent et sa dynamique jouent ainsi un rôle important dans la configuration d'un PNR. Elle conditionne autant les normes et les valeurs partagées, que les contraintes auxquelles font face les acteurs.

Dans la lignée des propositions des approches néo-institutionnalistes, nous adhérons à l'idée qu'il est nécessaire de distinguer dispositifs institutionnels en tant qu'espaces de négociation, et le système de gouvernance de la coordination qui en découle et qui sera spécifique à chaque collectif. Ce système de gouvernance édicte 
Author-produced version of the article publishedithristophe BOSCHET, Tina RAMBONILAZA Revue d'Economie Régionale et Urbaine RERU, 2010, n³, p. 569-593

The original publication is available at http://reru.gretha.u-bordeaux4.fr/

doi : $10.3917 /$ reru.103.0569

les règles et les normes, les responsabilités et les rôles et les fait respecter. Sans faire pour autant l'apologie de l'auto-organisation comme principe de fonctionnement de l'action collective et la forme de rupture que peut occasionner la mise en place d'un dispositif institutionnel formel, nous pensons que la dynamique de la coordination locale faisant suite à l'adoption d'un tel dispositif procède d'un processus d'apprentissage collectif. Les règles et les normes, le partage des rôles et des responsabilités, etc. qui en résultent sont définis au sein de l'espace de négociation délimité par les spécificités du dispositif institutionnel qui les a initiées, mais ne sont pas dérivés de celui-ci. La négociation autour d'un projet de PNR va être l'occasion pour chaque acteur d'agir sur la structure des relations et la qualité des ressources sociales ainsi générées ou produites. La qualité de ces ressources sociales, sans conditionner pour autant la réussite du projet collectif qui s'engage, peut du moins garantir l'adhésion des acteurs. L'interprétation de la place des dispositifs institutionnels territoriaux ne peut pas par conséquent s'affranchir de l'analyse de la coordination du système d'acteurs qui prévalait sur le territoire en répondant aux questions suivantes : qui sont les acteurs impliqués et quelles sont les règles et les normes qui régissent leur coordination? Puis à l'analyse de l'évolution de ce système d'acteurs : en quoi les spécificités du dispositif envisagé ou le projet collectif mobilise et recompose les relations sociales? Observe-t-on des acteurs nouveaux qui prennent place dans les processus de décision locaux. À l'inverse, certains acteurs sont-ils affaiblis ou marginalisés, voire exclus du processus en choisissant d'en sortir ? Une approche des PNR en termes de réseaux sociaux soulève la question suivante : la constitution d'un PNR sera-t-il un moyen de renforcer le repli sur soi de certains types d'acteurs locaux ou plutôt l'occasion de créer des ponts avec d'autres réseaux d'acteurs au sein du territoire qui soit plus favorable pour le développement local? En effet, les réseaux (structures sociales stabilisées) au sein desquels les acteurs sont encastrés constituent les principaux vecteurs de la diffusion des modèles et des argumentaires qui fondent le projet collectif, et autant comme facteur « d'enrôlement » des acteurs, que comme facteurs de " conflits » entre acteurs. Le PNR en tant que projet (périmètre, actions, acteurs associés) est le fruit de négociation entre les acteurs quant à la forme future de leur coordination.

\subsection{Place et rôle des différents acteurs du développement local}

L'analyse des dispositifs institutionnels territoriaux ne peut pas être déconnectée des bouleversements politiques favorisés par le processus de décentralisation initié en France depuis les années 1980. L'engagement direct de la responsabilité des élus et la multiplication des actions publiques territoriales font des acteurs politiques des moteurs de la vie locale à tout point de vue. Ainsi, pour ce qui concerne le processus de négociation autour des projets de développement territoriaux, les caractéristiques sociologiques des acteurs publics amènent à ne pas écarter leur spécificité au moment des négociations, sans quoi l'explicitation de ce processus particulier de genèse du dispositif demeurerait incomplète. Une entrée par les acteurs plus que par les organisations institutionnelles pour l'analyse, devrait par conséquent opérer une distinction entre élus et techniciens et ce faisant, une dynamique de négociation des acteurs leaders (politiques) et une dynamique de régulation des acteurs de terrain (LAJARGE et ROUX, 2007). Les élus disposent souvent 


\section{Les mécuthpsraresdureduersiph \&fitha artigle publiselsedinx sociaux}

Revue d'Economie Régionale et Urbaine RERU, 2010, n`3, p. 569-593

The original publication is available at http://reru.gretha.u-bordeaux4.fr/

doi : $10.3917 /$ reru.103.0569

de pouvoir de négociation en relation avec leur compétence administrative, ce qui différencie souvent le grand élu (conseiller régional, départemental, député, président de communauté de communes), des "simples maires». Aux relations entretenues par les positions institutionnelles se superposent les affinités amicales et professionnelles. En revanche, pour les techniciens, d'autres motifs sont susceptibles d'être soulevés, comme la permanence de leurs relations avec d'autres acteurs du développement, ou de leur maîtrise de la problématique en termes de compétences, et ainsi bénéficier de la confiance que leur accordent les autres acteurs de terrain. Cette complexité de la construction territoriale inscrite dans deux catégories différentes d'acteurs peut expliquer la nécessité d'un opérateur de mise en relation des acteurs autour d'un acteur central-leader qu'ils désignent eux-mêmes pour incarner le projet collectif. La constitution d'une compétence technique à travers une vraie équipe de travail au quotidien permet en revanche de veiller à la réalisation des objectifs et de maintenir et d'entretenir le climat de confiance au sein du collectif d'acteurs. Cette deuxième mission n'est cependant pas réalisable qu'à la condition que les acteurs locaux concernés (entrepreneurs, agriculteurs, opérateurs touristiques, associations citoyennes) soient impliqués. L'équipe du PNR peut alors jouer ce rôle d'intermédiation au quotidien entre différents réseaux d'acteurs. La construction territoriale serait alors l'observation d'un chevauchement de deux réseaux d'acteurs : les communautés ${ }^{6}$ de pratique qui se constituent autour des techniciens, et le réseau des relations qui gravitent autour des élus.

Une telle description de la coordination territoriale au sein des PNR peut sembler plausible. Cependant, elle fait fi de l'existence d'un processus de négociation de sa place initié par chacun des acteurs et permise par l'horizontalité du mode de coordination territoriale. La distinction élu/technicien peut alors s'estomper. En effet, rien ne prédestine un élu plutôt qu'un technicien à occuper le rôle de centralisateur de pouvoir (concentration des ressources financières, cognitives, relationnelles et capacité à les redistribuer au sein de la communauté pour la réalisation de l'objectif collectif). En effet, la légitimité du leadership en matière de coordination territoriale s'exprime au travers de la volonté de se coordonner, mais aussi de la réussite des actions menées (BERNARD et al., 1997). Ce double impératif amène ainsi à se réinterroger sur les spécificités relationnelles du mécanisme de coordination à l'œuvre. L'identification du leadership (en tant que négociateur) sera alors à rechercher non pas dans les rapports de pouvoirs et d'interdépendances en termes de ressources, mais comme la résultante des choix partenariaux de l'ensemble des acteurs impliqués, étant donné la nature des problèmes à résoudre et leur permanence dans le contexte territorial.

Nous formulons alors l'hypothèse que la configuration relationnelle des réseaux d'action publique locale est la résultante d'un processus itératif d'apprentissage collectif, face aux dilemmes sociaux auxquels les acteurs sont confrontés (fiabilité des partenaires, rapports de pouvoir et d'autorité du fait du jeu institutionnel). La pertinence ou l'originalité d'un dispositif institutionnel territorial réside par conséquent dans sa capacité à encadrer de manière formelle le processus de négociation autour du nouveau projet collectif, en offrant à chaque acteur une 
Author-produced version of the article published ihristophe BOSCHET, Tina RAMBONILAZA Revue d'Economie Régionale et Urbaine RERU, 2010, n³, p. 569-593

The original publication is available at http://reru.gretha.u-bordeaux4.fr/

doi : 10.3917/reru.103.0569

occasion d'agir sur les structures sociales définies par les participants du système d'acteurs en place et les mécanismes de coordination qui le gouvernent.

\section{-6 -}

\section{Remarques conclusives : intérêts et défis d'une approche en termes de réseaux sociaux de la dynamique territoriale}

A travers cet article, nous avons voulu circonscrire les apports et les outils de l'approche en termes de réseaux sociaux pour l'analyse de la dynamique territoriale centrée sur l'analyse de la coordination entre acteurs. Au sein de cette vaste littérature sur les réseaux, l'analyse de la dynamique territoriale prend essentiellement appui sur la compréhension de la dynamique des réseaux au travers de l'analyse de la formation stratégique des liens. La formalisation stratégique de la décision de chaque acteur, inséré et évoluant au sein d'un système social donné, permet de considérer que l'acteur est un entrepreneur social de son propre réseau. Le problème de coordination (les dilemmes sociaux inhérents à l'action collective) est donc géré de manière décentralisée (au niveau individuel) pour déboucher sur l'émergence endogène de processus de régulation social habituel (confiance, normes, identité). Chaque acteur, conscient de l'efficacité relative de chaque mécanisme de coordination, va tenter d'influencer son émergence ou son maintien au travers de son choix partenarial. Dans ce contexte, les dispositifs institutionnels territoriaux se proposent pour chaque acteur comme un espace de négociation de sa place au sein du système d'acteurs actuel. Ils édictent les règles du jeu de cette négociation, pour faciliter l'émergence ou le maintien d'un mécanisme de coordination spécifique.

Plusieurs interrogations tirent alors leurs réponses de l'analyse de la dynamique de la coordination territoriale dont quatre nous semblent importantes :

Quel est le mécanisme de coordination à l'œuvre dans les territoires ruraux ? En d'autres termes, quels sont les véritables dilemmes sociaux auxquels les acteurs font face dans la définition et la réalisation de projet de développement local fondé sur une valorisation de ce qui doit être reconnu comme principal atout du territoire et nécessite une vision partagée?

Dans quelle mesure les modèles de décisions locaux fondés sur le principe de participation d'un échantillon représentatif d'acteurs (syndicat mixte, comité de développement, comité de pilotage, etc..) organisent une mise en proximité entre ceux-ci et débouchent sur l'établissement de liens stables et utiles pour la concrétisation du projet collectif?

La configuration des relations entre les acteurs au sein des réseaux est-elle stable et permanente en l'absence de l'intervention d'un dispositif institutionnel formel? 


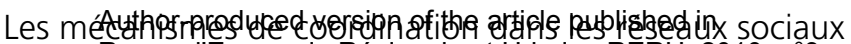

Revue d'Economie Régionale et Urbaine RERU, 2010, n`3, p. 569-593

The original publication is available at http://reru.gretha.u-bordeaux4.fr/

doi : $10.3917 /$ reru. 103.0569

Certains territoires institutionnels sont-ils plus importants que d'autres en dotant les acteurs qui leur sont rattachés, de ressources plus importantes, et qui permettent à ceux-ci d'occuper une place d'acteurs-clés dans la coordination du collectif ?

La principale difficulté liée à la mise en ouvre d'une approche en termes de réseaux sociaux de cette coordination territoriale réside dans sa mise en œuvre empirique. Comment disposer de données de relations adéquates et traduire ces données en véritables liens pertinents pour l'analyse. Ces liens doivent, en effet, traduire à la fois l'histoire des relations entre les individus et les collaborations économiques qui s'engagent entre ceux-ci. Dans un contexte d'explosion de l'approche en termes de réseaux sociaux des phénomènes économiques, la question sur l'intérêt d'une telle approche pour comprendre la morphogenèse des dispositifs institutionnels et la recomposition territoriale ne doit plus se poser. En revanche, la difficulté et le nécessaire arbitrage en termes de moyens d'investigation/production de connaissances qu'il s'agit d'effectuer constitue la principale limite de cette approche.

\section{Bibliographie}

ANGEON V., 2008, " L'explicitation du rôle des relations sociales dans les mécanismes de développement territorial ", Revue d'Economie Régionale et Urbaine, n², pp. 237-250.

Bala V., GoYal S., 2000, "A noncooperative model of network formation", Econometrica, vol. 68, n5, pp. 1181-1231.

Baraize F., Genyeis W., Faure A., Negrier E., Smith A., 2000, "Le pouvoir local en débat. Pour une sociologie du rapport entre leadership et territoires ", Pôle Sud, n¹3, pp. 103-119.

Barthelemy D., Nieddu M., Vivien F.D., 2004, «Externalités ou production de patrimoines ? Les enseignements de travaux récents sur l'agriculture et l'environnement », Géographie, Economie, Société, vol. 6, n³, pp. 331-352.

BeRARDO R., SCHOlz T.J., 2008, "Self-organizing policy networks: Risk, partner selection and cooperation in estuaries", Networks in political science (NIPS), June 12-14, Harvard, Massachusetts. http://www.hks.harvard.edu/netgov/files/NIPS/Berardo-Scholz_Final.pdf.

Bernard P., TAlbot D., WAllet F., 1997, "Pouvoirs, proximités et apprentissages, une relecture des relations par la dynamique interaction/action», colloque Proximité et coordination économique, Mai, Creuset, Lyon.

BILLET P., 2006, "Les parcs naturels régionaux aux risques des territoires", Revue Juridique de l'Environnement, $\mathrm{n}^{\circ}$ spécial, pp. 11-28.

Bramoullé Y., Fortin B., 2009, "The econometrics of social networks", in S.N. DurLaUf, et L.E. BLUME (eds.), New Palgrave Dictionnary of Economics.

Brousseau E., 2000, "La gouvernance des processus de coopération », in B. Bellon, C. VoISIN, A. PLUNKet (eds.), Coopération Industrielle : Synthèse et Diversité, Economica, Paris.

Brousseau E., 2001, "Confiance ou contrat, confiance et contrat », in F. AuberT et J-P. SYLVESTRE (eds.), Confiance et Rationalité, Actes du colloque UMR INRA/ENESAD, Dijon : 5 et 6 mai 1999, Inra Edition, Collection Les Colloques, Paris, pp. 65-80.

BURGer M.J., BUSKENS V., 2009, "Social context and network formation: An experimental study", Social Networks, vol.31, n¹, pp. 63-75.

BURT R.S., 1992, Structural holes: the social structure of competition, Harvard University Press, Cambridge, MA. 
Author-produced version of the article published ihristophe BOSCHET, Tina RAMBONILAZA Revue d'Economie Régionale et Urbaine RERU, 2010, n³, p. 569-593

The original publication is available at http://reru.gretha.u-bordeaux4.fr/ doi : $10.3917 /$ reru.103.0569

BURT R.S., 2004, "Structural holes and good ideas", American Journal of Sociology, vol. 110, $\mathrm{n}^{\circ} 2$, pp.349-399.

CAllois J.M., 2007, " Les limites du territoire : une application de la notion de rayon de confiance au développement territorial », Revue d'Economie Régionale et Urbaine, n5 5, pp. 811-830.

Cohendet P., Kirman A.P., Zimmerman J-B., 2003, «Emergence, formation et dynamique des réseaux, modèles de la morphogenèse ", Revue $d^{\prime}$ Economie Industrielle, $n^{\circ} 103$, pp. 15-42.

COLE A., JOHN P., "Les réseaux de politique publique en France et en Grande-Bretagne », in P. LE GALES, M. THATCHER, Les réseaux de politique publique. Débat autour des policy networks, L'Harmattan, Paris.

COLEMAN J.S., 1988, "Social capital in the creation of human capital", American Journal of Sociology, n 94 , pp. 95-120.

Coleman J.S., 1990, Foundations of social theory, Cambridge University Press.

Colletis G., Gilly J-P., PeCQueur B., 2001, «Inscription spatiale des firmes, gouvernance des territoires et régulation d'ensemble ", Troisièmes journées de la proximité, 13-14 décembre, Paris.

COLLETIS-WAHL K., 2008, " Micro-institutions et proximités: quelles lectures des dynamiques territoriales », Revue d'Economie Régionale et Urbaine, $\mathrm{n}^{\circ}$ 2, pp. 251-264.

DASGUPTA P., 2003, "Social capital and economic performance analytics", in E. Ostrom et T.K. AHN (eds.), Critical Studies in Economic Institutions Foundations of Social Capital, Edward Elgar.

Deroian F., 2003, "Le dilemme stabilité-efficacité dans les réseaux stratégiques : quelques éléments de littérature ", Revue d'Economie Industrielle, vol.103, n¹, pp 155-174.

DowDING K., 2001, "There must be end to confusion: Policy networks, intellectual fatigue, and the need for political science methods courses in British universities", Political Studies, vol. 43, n¹, pp. 136-158.

Durlauf S., Fafchamps M., 2005, "Social capital", in P. Aghion, et S. Durlauf (eds.), Handbook of Economic Growth, Elsevier, vol. 1, chapt.26, pp. 1639-1699.

DutTA B., VAN Den Nouweland A., TIJS S., 1998, "Link formation in cooperative situations", International Journal of Game Theory, vol. 27, n², pp. 245-256.

FALK A., KOSFELD M., 2003, "It's all about connections: Evidence on network formation", IEW Working Paper no. 146, Institute for Empirical Research in Economics, University of Zurich.

DE Federico De LA RUA A., 2004, "L'analyse longitudinale de réseaux sociaux totaux avec Siena. Méthode, discussion et application ", Bulletin de Méthodologie Sociologique, n 84, pp. 5-39.

FERRAND A., 1997, "La structure des systèmes de relations ", L'Année Sociologique, vol. 47, nº1, pp. 37-54.

Ferrand A., De Federico De la Rua A., 2006, "Methods of social networks analysis", in G. Casella, J. VAluIN et G. WUNSCH (eds.), Demography: Analysis and Synthesis, Academic Press.

FerRaRY M., DibiagGiO L., 2003, "Communautés de pratique et réseaux sociaux dans la dynamique de fonctionnement des clusters de hautes technologies ", Revue $d^{\prime}$ Economie Industrielle, $\mathrm{n}^{\circ} 103, \mathrm{p}$. $111-130$.

FORSÉ M., 2008, "Définir et analyser les réseaux sociaux », Informations Sociales, vol. 3, n ${ }^{\circ}$ 147, pp. 10-19.

FreEMAN L.C, 1979, "Centrality in social networks, conceptual clarification”, Social Networks, vol. 1, n³, pp. 215-239.

Gilly J.P, WALLET F., 2005, "Enchevêtrement des espaces de régulation et gouvernance territoriale », Revue d'Economie Régionale et Urbaine, n5, pp. 699-722.

Goyal S., VeGA-RedOndo F., 2005, "Network formation and social coordination", Games and Economic Behavior, vol. 50, $\mathrm{n}^{\circ} 2$, pp. 178-207.

Granovetter M., 1973, "The strength of weak ties", American Journal of Sociology, vol. 78, n6, pp. 1360-1380.

Granovetter, M., 1985, "Economic action and social structure: The problem of embededdness", American Journal of Sociology, vol. 91, n³, pp. 481-510. 


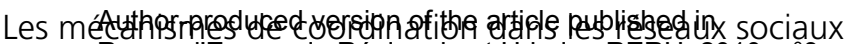

Revue d'Economie Régionale et Urbaine RERU, 2010, n`3, p. 569-593

The original publication is available at http://reru.gretha.u-bordeaux4.fr/

doi : $10.3917 /$ reru.103.0569

Granovetter M., 2000, Le Marché Autrement, Desclée De Brouwer, Collection Sociologie économique, Paris.

Hassenteufel P., 2008, Sociologie politique : I'action publique, Armand Colin, Collection « U », Paris.

HeCKATHORN D.D., 1996, "The Dynamics and dilemmas of collective action", American Sociological Review, vol. 71, n², pp. 235-259.

HOLLAND P.W., LeIHNARDT S., 1976, "The statistical analysis of local structures in social networks", in D.R. HeISE (eds.), Sociological Methodology, Josey Bass, San Francisco, pp. 1-45.

JACKSON M.O., WOLINSKY A., 1996, "A Strategic model of social and economic networks", Journal of Economic Theory, vol. 71, $\mathrm{n}^{\circ} 1$, pp.44-74.

JACKSON M.O., WATTS A., 2002, "On the formation of interaction networks in social coordination games", Games and Economic Behavior, vol. 41, n², pp. 265-291.

KENIS P., RAAB J., 2003, "Wanted: A good network theory of policy making", $7^{\text {th }}$ National Public Management Conference, October 9-10, Washington D.C.

KOSFELD M., 2004, "Economic networks in the laboratory, a survey", Review of Network Economics, vol. $3, \mathrm{n}^{\circ} 1$, pp. 20-41.

KREPS D., 1990, Game Theory and Economic Modelling, Oxford University Press, New-York.

LAJARGE R., RouX E., 2007, "Ressource, projet, territoire : le travail continu des intentionnalités », in $\mathrm{H}$. GumuChIAN, B. PECQUeUR, Les ressources territoriales, Economica, Paris, pp. 133-146.

Lallement M., 2006, "Capital social et théories sociologiques », in A. Bevort et M. Lallement (eds.), Le capital social. Performance, équité et réciprocité, La Découverte, Paris, pp.71-88.

LEAVIT H., 1951, "Some effects of certain communication patterns on group performance", Journal of Abnormal and Social Psychology, vol. 46, pp. 38-50.

Le Gales P., Thatcher M., 1995, Les réseaux de politique publique. Débat autour des policy networks, L'Harmattan, Paris.

Lemieux V., Ouimet M., 2004, L'analyse structurale des réseaux sociaux, De Boeck, Collection Méthodes en sciences humaines.

MARWell G., Oliver P.E., 1993, The critical mass in collective action: A micro social theory, Cambridge University Press.

MENARD C., 2003, "L'approche néo-institutionnelle : des concepts, une méthode, des résultats ", Cahiers $d^{\prime}$ Economie Politique, $\mathrm{n}^{\circ}$ 44, pp. 103-118.

NORTH D.C., 1990, Institutions, institutional change and economic performance, Cambridge University Press.

OLIVER P.E., 1980, "Rewards and punishments as selective incentives for collective action: Theoretical investigations", American Journal of Sociology, n 85, pp. 1356-1375.

OLSON M., 1965, The logic of collective action: Public goods and the theory of groups, Harvard University Press.

Ostrom E., 1990, Governing the commons: The evolution of institutions for collective action, Cambridge University Press.

Ostrom E., 1992, "Policy analysis of collective action and self-governance", Annual Policy Review, vol. 10, pp. 81-119.

Ostrom E., 1998, "Self-governance of common pool resources", in P. Newman, The New Palgrave Dictionary of Economics and the Law, pp. 424-433.

Ostrom E., 2000, "Collective action and the evolution of social norms", Journal of Economic Perspectives, vol. 14, n³, pp. 137-158.

PADGETT J.F., ANSELL C., 1993, "Robust action and the rise of the Medici, 1400-1434", American Journal of Sociology, vol. 98, n6, pp. 1259-1319.

Pecqueur B, Zimmermann J.B., 2004, Economie de proximités, Hermès Lavoisier, Paris.

PONTHIEUX S., 2006, Le capital social, La Découverte, Collection Repères, Paris. 
Author-produced version of the article published his ristophe BOSCHET, Tina RAMBONILAZA Revue d'Economie Régionale et Urbaine RERU, 2010, n³, p. 569-593

The original publication is available at http://reru.gretha.u-bordeaux4.fr/ doi : $10.3917 /$ reru.103.0569

ROBINS G., PATTISON P.E., WASSERMAN S., 1999, "Logit models and logistic regressions for social networks. III. Valued relations", Psychometrika, vol. 64, n³, pp. 371-394.

ROBINS G., PATTISON P.E., KALISH Y., LUSHER D., 2007, "An introduction to exponential random graph ( $p$ *) models for social network", Social Networks, vol. 29, n², pp.173-191.

SNIIDERS T.A.B., VAN DE BUNT G.G., STEGLICH C.E.G., 2009, "Introduction to stochastic actor-based models for network dynamics", Social Networks, vol. 32, $n^{\circ} 1$, pp. 44-60.

Ternaux P., 2007, "Dynamiques territoriales, structures sociales et comportement des acteurs", XLII èm e Colloque de I'ASRDLF, Grenoble-Chambéry, 11-13 juillet.

WASSERMAN S., 1980, "Analyzing social networks as stochastic processes", Journal of American Statistical Association, vol. 75, n³70, pp. 280-294.

WASSERMAN S., FAUST K., 1994, Social network analysis, methods and applications, Cambridge University Press.

WASSERMAN S., PATTISON P.E., 1996, "Logit models and logistic regression for social networks: An introduction to MARKOV graphs and $\mathrm{p}^{* \prime \prime}$, Psychometrika, vol. 61, n 3 , pp. 401-425.

Wenger E.R., MCDermott R., SNyder W.M., 2002, Cultivating communities of practice: A guide to managing knowledge, Harvard Business School Press, Boston.

Williamson O.E., 1985, The economic institutions of capitalism, The Free Press, New York.

\section{Notes}

1 - La notion de transactions économiques est assimilée ici à la coopération, « la coopération comme catégorie particulière de transaction » (BROUSSEAU, 2001).

2 - Toutes les citations non datées de cet auteur font référence à BuRT (1992) indexé en bibliographie.

3 - On utilise "structural " quand il s'agit de parler des propriétés du réseau en tant que graphe, et "structurel » lorsque l'on s'intéresse aux liens et aux relations.

4 - À partir du moment où il est accepté que, par exemple, l'établissement d'une relation entre deux individus peut-être le fruit de l'influence d'une troisième personne qui a déjà un lien avec eux.

5 - Dans certains PNR, la nécessité de poursuivre au niveau communal l'inscription dans les plans locaux d'urbanisme de mesures de maîtrises foncières afin de limiter le mitage de l'espace et la dégradation des paysages n'a pas été poursuivie par certaines communes signataires, pour des raisons fiscales ou pour attirer de nouveaux résidents.

6 - «Les communautés de pratique sont des groupes d'individus partageant le même intérêt, le même ensemble de problèmes, ou une passion autour d'un thème spécifique et qui approfondissent leur connaissance et leur expertise en interagissant régulièrement ", (WenGer et al. 2002, page 4 citée par FerRARY et Dibiaggio (2003)). 